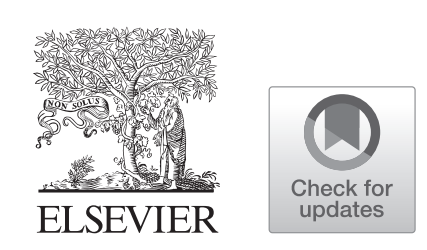

\title{
Drug-Eluting Stent Shows Similar Patency Results as Prosthetic Bypass in Patients with Femoropopliteal Occlusion in a Randomized Trial
}

\author{
Patrick Björkman, ${ }^{1}$ Tommi Auvinen, ${ }^{2}$ Harri Hakovirta, ${ }^{3}$ Pekka Romsi, ${ }^{4}$ Johanna Turtiainen, \\ Hannu Manninen, ${ }^{6}$ and Maarit Venermo, ${ }^{1}$ Helsinki, Kuopio, Turku, Oulu, and Joensuu, \\ Finland
}

Background: Claudication and critical limb threatening ischemia are significant causes of mortality in the elderly. The gold standard of superficial femoral artery (SFA) revascularization is thus far considered to be the femoropopliteal bypass. The aim of this study was to compare mid-term patency between drug-eluting stents (DESs) and prosthetic bypass grafts (BSX). Studies have reported comparable results for both the methods.

Methods: Forty-six patients with claudication or rest pain due to a 5-25 cm SFA occlusion were randomized between DES and BSX groups. The follow-up period was 24 months, and the primary outcome measure was overall patency. Secondary outcome measures were primary and primary assisted patency, change in ankle-brachial index (ABI), and amputation-free survival.

Results: Forty-one patients were eventually analyzed. Six-month secondary patency was $91 \%$ (DES) versus $83 \%$ (BSX) $(P=0.450)$. The corresponding numbers at 12 months in the DES and BSX groups were $74 \%$ and $80 \%(P=0.750)$, respectively. At 24 months, the respective numbers were $56 \%$ and $71 \%(P=0.830)$. There were no statistically significant differences in primary or assisted primary patency at 1,6 , or 12 months.

Conclusion: There were no demonstrable differences in patency rates or clinical outcomes such as $\mathrm{ABI}$ or major amputations between DES and BSX. Although underpowered, the results suggest noninferiority of the DES compared with prosthetic bypass surgery. Trial registration: The trial was preregistered at ClinicalTrials.org (NCT01450722).

The authors H.M. and M.V. shared last authorship.

The FinnPTX-study group: Kimmo Lappalainen, MD, Helsinki University Hospital, Department of Radiology; Anders Albäck, $M D$ PhD, Helsinki University Hospital, Department of Vascular Surgery; Antti Korpela, MD, Lahti Central Hospital, Department of Surgery; Kimmo Mäkinen, MD, PhD, Kuopio University Hospital, Department of Vascular Surgery; Petri Saari, MD, PhD, Kuopio University Hospital, Department of Radiology; Jukka Perälä, MD, PhD, Oulu University Hospital, Department of Radiology.

The trial received a grant from the Finnish Society of Interventional Radiology.

${ }^{I}$ Department of Vascular Surgery and University of Helsinki, Helsinki University Hospital, Helsinki, Finland.

${ }^{2}$ Department of Vascular Surgery, Kuopio University Hospital, Kuopio, Finland.

${ }^{3}$ Department of Vascular Surgery, Turku University Hospital, Turku, Finland.
${ }^{4}$ Department of Vascular Surgery, Oulu University Hospital, Oulu, Finland.

${ }^{5}$ Department of Surgery, Joensuu Central Hospital, Joensuu, Finland.

${ }^{6}$ Department of Radiology, Kuopio University Hospital, Kuopio, Finland.

Correspondence to: Patrick Björkman, MD, Department of Vascular Surgery and University of Helsinki, Helsinki University Hospital, Helsinki, Finland; E-mail: patrick.bjorkman@hus.fi

Ann Vasc Surg 2018; 53: 165-170

https://doi.org/10.1016/j.avsg.2018.04.014

(c) 2018 Elsevier Inc. All rights reserved.

Manuscript received: February 28, 2018; manuscript accepted: April 10, 2018; published online: 7 June 2018 


\section{INTRODUCTION}

Critical limb threatening ischemia (CLTI) due to atherosclerosis causes significant morbidity especially in the elderly, and if untreated, it leads to limb loss. ${ }^{1}$ The incidence of CLTI is estimated at 500-1,000/million annually. ${ }^{2}$ The prevalence of its milder symptomatic manifestation, intermittent claudication, among men aged 40-44 years is about $0.4 \%$ and $3 \%$ among men aged 65-69 years. $^{3}$ In asymptomatic peripheral artery disease or claudication, the ischemia relatively rarely deepens over time to threaten the vitality of the limb. ${ }^{4}$ Although intermittent claudication has a benign prognosis and can often be treated conservatively, more severe forms with extensive arterial obstructions and symptoms that impair the quality of life significantly require revascularization. Open bypass surgery (BSX) is currently considered the gold standard for treating long obstructions in the superficial femoral artery (SFA). Up to $81 \%$ of 2-year patency can be appreciated after bypass with a good-quality saphenous vein. If synthetic graft is used, the long-term patency is somewhat lower, which is up to $67 \%$ according to a systematic review. ${ }^{5}$

Treatment of femoropopliteal occlusive disease has shifted dramatically toward endovascular methods during the last 10 years. ${ }^{6}$ Despite this, superiority of endovascular treatment has not been definitively demonstrated. In 2010, the BASIL trial concluded that in patients with severe ischemia, femoropopliteal BSX with a vein graft was superior to primary balloon angioplasty (BA), but that primary BA was superior to prosthetic BSX. $^{7}$ Furthermore, it was concluded that failed BA yielded worse outcomes for future ipsilateral BSX. This has been supported by subsequent studies. ${ }^{8}$ Comparison of stenting and prosthetic bypass grafting has yielded similar results at 4 -year follow-up in an randomized controlled trial with 100 revascularizations and in a smaller, retrospective study. 9,10

Drug-eluting stents (DESs) have proven their worth in coronary artery lesions and to some degree in femoral occlusions. ${ }^{11}$ Although early trials failed to show benefit from DES versus bare metal stents, ${ }^{12,13}$ subsequent studies have shown improved event-free survival up to 5 years. ${ }^{14}$ Paclitaxel is a mitotic inhibitor and an antiproliferative agent. ${ }^{15}$ It is a widely used, effective agent in drug-eluting stents to reduce restenosis in coronary circulation. ${ }^{16}$ Paclitaxel binds specifically to the beta-tubulin subunit of microtubules and appears to antagonize the disassembly of this key cytoskeletal protein; this action results in accumulation of microtubule bundles and aberrant microtubule-derived structures in the mitotic phase of the cell cycle. ${ }^{17,18}$ The Zilver PTX (Cook Medical) paclitaxel-eluting stent is designed specifically for use in the SFA. It is a nitinol stent coated with paclitaxel only, without a polymer or binder.

This trial is a prospective, randomized, multicenter trial comparing outcomes for prosthetic above-knee (AK) bypass versus the Zilver PTX drug-eluting stent. Bypass with a synthetic graft, instead of autogenous vein, is used as a reference standard because of the difficulty to standardize the quality of available vein and because bypasses to the proximal popliteal artery with the different graft types give comparable results. The trial was investigator initiated and did not receive funding from the industry.

\section{MATERIALS AND METHODS}

Patients were randomized at 6 hospitals in Finland (Helsinki, Oulu, Turku, and Kuopio University hospitals and the central hospitals in Lahti and Joensuu). Patients were included between 2011 and 2014, and the follow-up ended in 2016. Patients presented with rest pain or severe claudication (Rutherford class II-IV), and patients with wounds or tissue loss were excluded. SFA lesions of $5-25 \mathrm{~cm}$ were eligible for inclusion. The lesions were diagnosed and measured using magnetic resonance angiography or computed tomography angiography. Concomitant inflow or outflow procedures were not allowed. All patients provided written informed consent. Inclusion and exclusion criteria are listed in Table I. Patients were randomized to BA+DES or prosthetic AK femoropopliteal bypass groups. A block randomization of 2:1 (DES:BSX) was performed at the ward or outpatient clinic after checking eligibility and receiving signed informed consent.

\section{Bypass Surgery}

Bypass surgery was performed under general anesthesia or spinal blockade from incisions to the groin and proximal popliteal artery. A 6-mm heparin-bonded polytetrafluoroethylene (PTFE) graft was used. The graft was tunneled anatomically or subcutaneously depending on surgeon's preference. Procedures were performed under systemic heparinization with an activated clotting time between 200 and 300 sec. 
Table I. Inclusion and exclusion criteria.

\begin{tabular}{ll}
\hline Inclusion criteria & Exclusion criteria \\
\hline Rutherford class II-IV & $\begin{array}{c}\text { Previous treatment for } \\
\text { same lesion }\end{array}$ \\
SFA occlusion of $5-25 \mathrm{~cm}$ & $\begin{array}{c}\text { Indication for } \\
\text { infrapopliteal treatment } \\
\text { Iodine allergy }\end{array}$ \\
$\begin{array}{c}\text { Eligible for operative } \\
\text { treatment }\end{array}$ & $\begin{array}{c}\text { Patients undergoing } \\
\text { heast l vessel crural } \\
\text { runoff }\end{array}$ \\
$\begin{array}{c}\text { Written informed consent } \\
\text { Pregnancy }\end{array}$ \\
\hline
\end{tabular}

\section{Balloon Angioplasty and DES}

Access was obtained from the ipsilateral or contralateral common femoral artery. The occlusion was recanalized and crossed intraluminally or subintimally before predilatation and stent deployment. The stent was postdilated according to instructions for use. Patients received 5,000 IU systemic heparin during the procedure.

\section{Follow-up and Outcome Measures}

The follow-up period was 24 months, and the primary outcome measure was overall stent or graft patency. Secondary outcome measures were primary and assisted patency, change in anklebrachial index $(\mathrm{ABI})$, and amputation-free survival. The follow-up comprised clinical evaluation for symptoms and duplex ultrasound to assess patency at $1,6,12$, and 24 months, postoperatively.

\section{Antithrombotic Regime}

Postoperatively, all patients except those on warfarin were started on life-long American Society of Anesthesiologists (ASA) treatment in both the treatment groups. Patients in the DES group were on dual antiplatelet therapy (ASA $100 \mathrm{mg}$ + clopidogrel $75 \mathrm{mg}$ daily) for at least 3 months postoperatively. DES patients on warfarin were started on low-dose $(50 \mathrm{mg}$ ) ASA for the same period. Dual antiplatelet therapy was not prescribed after bypass surgery.

\section{Randomization}

Block randomization (2:1) was performed by concealed envelope by the research nurse at the University of Kuopio. Owing to the nature of the study, all subjects, providers, and outcomes assessors were not blinded.

\section{Statistical Analysis}

A statistical analysis was performed using the SPSS v. 22 software (IBM, Armonk, VA). Continuous variables are expressed as means and range or medians and interquartile range, and dichotomous variables are expressed as percentages. Continuous variables were compared using Mann-Whitney test and dichotomous variables using chi-squared test. Patency rates were analyzed with Log-rank testing.

The study was approved by the ethical boards of Kuopio University Hospital and Helsinki University Hospital, and the study design was declared and preregistered at ClinicalTrials.org (NCT01450722).

\section{RESULTS}

Forty-six patients were randomized. Baseline characteristics are described in Table II. Five patients were excluded due to immediate technical failure, that is, unsuccessful recanalization. These were salvaged by distal and/or venous bypass, and thus they were not eligible for the intention-to-treat analysis. There were no deaths or major amputations in either group during the 12-month followup; 1 patient in the stent group died at 24 months from procedure due to unrelated disease. The number of patients lost to follow-up at 6,12 , and 24 months was $0(0.0 \%), 6(14.2 \%)$, and 11 $(26.2 \%)$, respectively. In the DES group, the median number of stents was 2 (range, $1-4$ ) with a median diameter of $6 \mathrm{~mm}$.

Forty-one patients were eventually analyzed. Six-month primary patency was $82.6 \%$ (DES) versus $72.2 \%(\mathrm{BSX}) \quad(P=0.447)$ and secondary patency was $91 \%$ versus $83 \%(P=0.450)$. The 12 month secondary patency in the DES and BSX groups was $74 \%$ compared to $80 \%(P=0.750)$, respectively. There were no statistically significant differences in primary, assisted primary, or secondary patency at $1,6,12$, or 24 months (Table III; Figs. 1 and 2). The median ABI rose from 0.54 to 0.93 in the DES group and from 0.65 to 1.02 in the BSX group after the procedures, and there were no significant differences between the groups neither at the baseline nor during the follow-up (Table IV). Relative risk for stenting at 1 year was 0.96 $(P=0.893$, any endpoint $)$.

\section{DISCUSSION}

In the current trial, no significant differences between femoropopliteal AK bypass with PTFE prosthesis and endovascular recanalization and stenting with Zilver PTX stenting could be 
Table II. Baseline characteristics

\begin{tabular}{|c|c|c|c|c|c|}
\hline & DES $(n=23)$ & Range/Mean & $\operatorname{BSX}(n=18)$ & Range/Mean & $P$ value \\
\hline Age & 68 & $48-88$ & 67 & $50-84$ & 0.398 \\
\hline Male sex & 17 & & 12 & & 0.613 \\
\hline Tl diabetes & 6 & 26.1 & 4 & 22.2 & 0.775 \\
\hline $\mathrm{T} 2$ diabetes & 3 & 13.0 & 2 & 11.1 & 0.650 \\
\hline Smoking & 9 & 39.1 & 6 & 33.3 & 0.702 \\
\hline Ex-smoker & 9 & 39.1 & 5 & 27.8 & 0.230 \\
\hline $\begin{array}{l}\text { Transient ischemic } \\
\text { attack (TIA) }\end{array}$ & 2 & 8.7 & 2 & 11.1 & 0.796 \\
\hline Stroke & 3 & 13.0 & 2 & 11.1 & 0.851 \\
\hline Coronary disease & 6 & 26.1 & 5 & 27.8 & 0.903 \\
\hline Prior acute myocardial infarction & 1 & 4.3 & 3 & 16.7 & 0.187 \\
\hline Dyslipidemia & 13 & 56.5 & 15 & 83.3 & 0.067 \\
\hline Chronic heart disease & 2 & 8.7 & 2 & 11.1 & 0.796 \\
\hline Hypertension & 15 & 65.2 & 15 & 83.3 & 0.194 \\
\hline Pulmonary & 1 & 4.5 & 1 & 5.6 & 0.884 \\
\hline ASA & 21 & 91.3 & 14 & 77.8 & 0.224 \\
\hline Clopidogrel & 1 & 4.3 & 4 & 22.2 & 0.083 \\
\hline Warfarin & 3 & 13.0 & 2 & 11.1 & 0.851 \\
\hline Other & 2 & 8.6 & 1 & 5.6 & 0.653 \\
\hline Statin therapy & 12 & 52.2 & 14 & 77.8 & 0.051 \\
\hline $\begin{array}{l}\text { Angiotensin-converting-enzyme/ } \\
\text { angiotensin II-blockade }\end{array}$ & 9 & 39.1 & 9 & 50.0 & 0.656 \\
\hline ABI (mean) & 0.54 & $0-0.82$ & 0.65 & $0.47-0.99$ & 0.120 \\
\hline SFA occlusion length (mm) & 13.2 & $5.0-25.0$ (IQR 12.3) & 11.3 & $5.0-19.6(\mathrm{IQR} 7.9)$ & 0.424 \\
\hline \multicolumn{6}{|l|}{ Rutherford classification } \\
\hline I & 4 & 17.4 & 3 & 16.7 & 0.359 \\
\hline II & 7 & 30.4 & 8 & 44.4 & \\
\hline III & 6 & 26.1 & 6 & 33.3 & \\
\hline IV & 6 & 26.1 & $\mathrm{l}$ & 5.6 & \\
\hline \multicolumn{6}{|l|}{ Crural runoff* } \\
\hline 3 & 8 & 34.8 & 5 & 27.8 & 0.782 \\
\hline 2 & 8 & 34.8 & 4 & 22.2 & \\
\hline 1 & 7 & 30.4 & 2 & 11.1 & \\
\hline 0 & 0 & 0 & 1 & 5.6 & \\
\hline
\end{tabular}

*Missing data in the BSX group.

demonstrated. At 6 months, the primary and secondary patencies were slightly, but not significantly, higher in the stent group than those in the bypass group, but this difference disappeared during the next 6 months. At 12 months, the respective rates were surprisingly similar: $63.2 \%$ versus $66.7 \%$ and $74 \%$ versus $80 \%$. Indeed, at 2 years, the patency rate was better in the BSX group (56\% vs. $71 \%$, $P=0.397$ ), but at this stage, the number at risk is substantially lower than that at the earlier followups.

For the time being, open femoropopliteal BSX is a first-hand option in many centers worldwide. Use of prosthetic grafts for AK bypasses remains popular because of many surgeons' preference to save the saphenous veins for possible future below-knee or distal bypasses and speed of the procedure. The Zilver PTX DES is designed specifically for femoropopliteal locations. A prospective, randomized trial reported significantly better 24-month event-free survival among patient receiving a DES than that among those treated with percutaneous transluminal angioplasty (PTA) alone $(86.6 \%$ vs. $77.6 \%, P<0.01) .{ }^{19}$ Primary patency at 24 months of the DES group was $74.8 \%$ versus $32.4 \%$ for the PTA group. Patency rates at 5 years further favored the Zilver PTX. ${ }^{14}$ The Zilver PTX trials have shown patency rates in the $80 \%$ range at 12 months, which are comparable to our results.

The Scandinavian Thrupass study demonstrated a clear benefit in favor of bypass surgery versus the Gore endoluminal PTFE thrupass. ${ }^{20}$ This trial showed a remarkable 95\% 1-year patency in the bypass treatment group, whereas the corresponding number was only $48 \%$ for the thrupass group. In 2007, Kedora et al. ${ }^{21}$ demonstrated comparable 
Table III. Primary, assisted primary, and secondary patency rates for BSX and DES

\begin{tabular}{llll}
\hline & & & $\begin{array}{l}P \text { value } \\
\text { (log rank) }\end{array}$ \\
\hline $1 \mathrm{~m}$ & & & \\
$\quad$ Primary patency (\%) & 87.0 & 88.9 & 0.872 \\
$\quad$ Assisted primary patency (\%) & 87.0 & 88.9 & 0.872 \\
$\quad$ Secondary patency (\%) & 87.0 & 94.4 & 0.454 \\
6 m & & & \\
$\quad$ Primary patency (\%) & 87.0 & 72.2 & 0.447 \\
$\quad$ Assisted primary patency (\%) & 91.3 & 77.8 & 0.247 \\
$\quad$ Secondary patency (\%) & 91.3 & 83.3 & 0.450 \\
12 m & & & \\
$\quad$ Primary patency (\%) & 63.2 & 66.7 & 0.931 \\
$\quad$ Assisted primary patency (\%) & 68.4 & 73.3 & 0.840 \\
$\quad$ Secondary patency (\%) & 73.7 & 80.0 & 0.750 \\
24 m & & & \\
$\quad$ Secondary patency (\%) & 56.3 & 71.4 & 0.830 \\
\hline
\end{tabular}

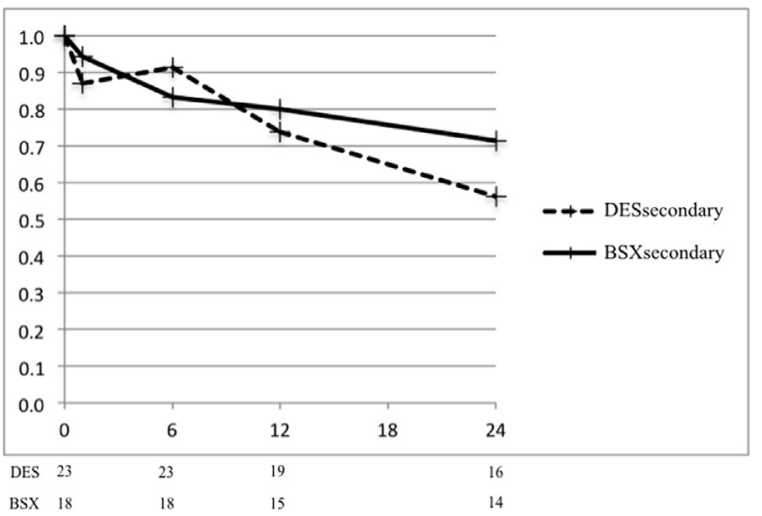

Fig. 1. 24-month secondary patency for BSX (solid line) and DES (dashed line).

1-year outcomes between the Viabahn covered stent (CS) and prosthetic AK femoropopliteal bypass. This study included 100 limbs in a prospective setting. In this study, 6- and 12-month patency rates were $82 \%$ (BSX) versus $81.8 \%$ (CS) and $73.5 \%$ versus $74.2 \%$, respectively. In our study, the patency rates were somewhat higher at 6 months and lower at 12 months but still in comparable figures. The study by Kedora et al. has been criticized for including Trans-Atlantic InterSociety Consensus A lesions.

It should be noted that 5 cases $(5 / 27,18.5 \%)$ were excluded from the DES group because of failed recanalization, whereas the primary technical success rate in the BSX group was $100 \%$. In l case, the attempted recanalization resulted in severe distal dissection and acute ischemia, which eventually could be salvaged with a distal bypass. We did not report the results for patients with technical

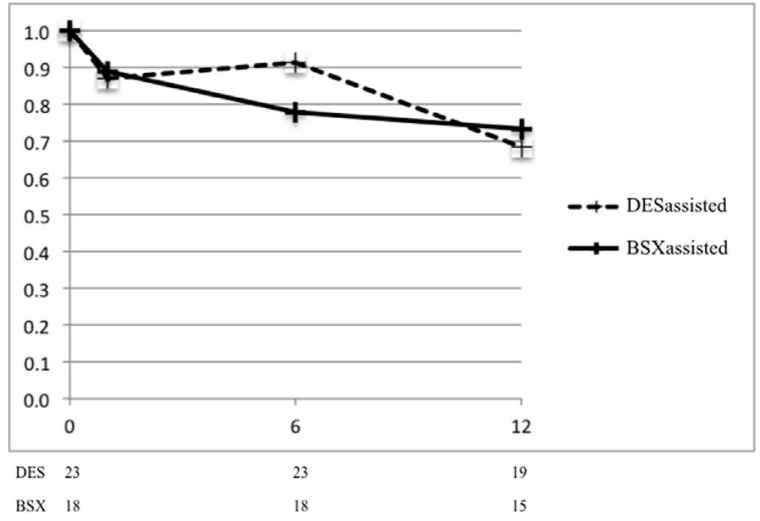

Fig. 2. 12-month assisted primary patency for BSX (solid line) and DES (dashed line).

Table IV. 12-month ABI for BSX and DES

\begin{tabular}{lllllll}
\hline & \multicolumn{2}{l}{ DES } & \multicolumn{2}{l}{ BSX } & \\
\cline { 2 - 3 } ABI & Mean & Range & & Mean & Range & \multirow{2}{*}{$P$ value } \\
\hline Postoperative & 0.93 & $0.63-1.38$ & & 1.02 & $0.76-1.42$ & 0.220 \\
$1 \mathrm{~m}$ & 0.99 & $0.39-1.85$ & & 0.94 & $0.78-1.09$ & 0.620 \\
$6 \mathrm{~m}$ & 0.93 & $0.59-2.00$ & 0.80 & $0.31-1.12$ & 0.650 \\
$12 \mathrm{~m}$ & 0.86 & $0.73-0.98$ & & 0.85 & $0.54-1.05$ & 0.791 \\
\hline
\end{tabular}

failures, but no statistically significant difference was seen in a sensitivity analysis including these patients. Furthermore, 1 case in the DES group received a bailout CS after perforation and hemorrhage. This did not compromise patency, as the DES in question was patent at 2 years.

In this trial, there was a significant difference between the groups in time from diagnosis to treatment. The time from CTA or MRA to treatment was 60 days in the DES group and 125 days in the BSX group $(P<0.01)$. This is likely due to hospital logistics and the more rigorous medical workup procedure before the bypass surgery. There was no evidence that this delay would have resulted in clinical deterioration in the BSX patients before surgery.

This trial is limited by the small sample size, and consequently there is a marked risk for type II error in the patency rates. The primary reason for the slow inclusion and randomization rate was the quickly somewhat ageing hypothesis and clinically problematic setting for prosthetic bypass surgery; few surgeons would end up including the shorter SFA lesions into this trial design, as these are routinely treated with less invasive endovascular procedures or, indeed, venous bypass grafting. This is overall seen in decreasing rates of open AK bypass surgery and quite the opposite in successful endovascular femoropopliteal revascularizations. 
Despite its limitations, we think our article gives valuable information on the outcome after these 2 procedures, and it seems that the DES is not inferior to prosthetic AK bypass in patients with SFA occlusion of $25 \mathrm{~cm}$ or less. This is the only prospective trial to date comparing DES with bypass surgery, and the results do indicate that drug-eluting stents are comparable to prosthetic grafts with regard to patency. Another strength of the trial is the comprehensive follow-up at 6 months and acceptable follow-up rates up to 24 months. In anticipation of larger trials, the results from this trial loosely favor endovascular revascularization and use of DES for SFA lesions if no vein is available for grafting.

\section{CONCLUSIONS}

This is the first randomized trial comparing the DES to prosthetic bypass in AK femoropopliteal occlusion. At 12 and 24 months after the procedure, there was no statistically significant difference in primary patency, assisted primary patency, or secondary patency between the groups. Although underpowered, our study suggests noninferiority of the DES compared to PTFE bypass in this patient group. Larger studies are needed for more definitive conclusions.

The trial was supported by a grant from the Finnish Society of Interventional Radiology.

\section{REFERENCES}

1. Wolfe J, Wyatt M. Critical and subcritical ischaemia. Eur J Vasc Endovasc Surg 1997;13:578-82.

2. Norgren L, Hiatt WR, Dormandy JA, et al. Inter-society consensus for the management of peripheral arterial disease (TASC II). Eur J Vasc Endovasc Surg 2007;33:S1-75.

3. Jensen SA, Vatten LJ, Romundstad PR, et al. The prevalence of intermittent claudication. Sex-related differences have been eliminated. Eur J Vasc Endovasc Surg 2003;25: 209-12.

4. Dormandy JA, Murray GD. Reprinted article "the fate of the claudicant-a prospective study of 1969 claudicants". Eur J Vasc Endovasc Surg 2011;42:S4-6.

5. Klinkert P, Post PN, Breslau PJ, et al. Saphenous vein versus PTFE for above-knee femoropopliteal bypass. A review of the literature. Eur J Vasc Endovasc Surg 2004;27:357-62.

6. Garg K, Kaszubski PA, Moridzadeh R, et al. Endovascularfirst approach is not associated with worse amputation-free survival in appropriately selected patients with critical limb ischemia. J Vasc Surg 2014;59:392-9.
7. Bradbury AW, Adam DJ, Bell J, et al. Bypass versus Angioplasty in Severe Ischaemia of the Leg (BASIL) trial: analysis of amputation free and overall survival by treatment received. J Vasc Surg 2010;51:18S-31S.

8. Nolan BW, De Martino RR, Stone DH, et al. Prior failed ipsilateral percutaneous endovascular intervention in patients with critical limb ischemia predicts poor outcome after lower extremity bypass. J Vasc Surg 201 1; 54:730-5.

9. McQuade K, Gable D, Pearl G, et al. Four-year randomized prospective comparison of percutaneous ePTFE/nitinol selfexpanding stent graft versus prosthetic femoral-popliteal bypass in the treatment of superficial femoral artery occlusive disease. J Vasc Surg 2010;52:584-90.

10. Linnakoski H, Uurto I, Suominen V, et al. Comparison of above-the-knee prosthetic femoro-popliteal bypass versus percutaneous transluminal angioplasty and stenting for treatment of occlusive superficial femoral artery disease. Scand J Surg 2013;102:227-33.

11. Windecker S, Remondino A, Eberli FR, et al. Sirolimuseluting and paclitaxel-eluting stents for coronary revascularization. N Engl J Med 2005;353:653-62.

12. Duda SH, Bosiers M, Lammer J, et al. Sirolimus-eluting versus bare nitinol stent for obstructive superficial femoral artery disease: the SIROCCO II trial. J Vasc Interv Radiol 2005;16:331-8.

13. Lammer J, Bosiers M, Zeller T, et al. First clinical trial of nitinol self-expanding everolimus-eluting stent implantation for peripheral arterial occlusive disease. J Vasc Surg 2011;54:394-401.

14. Dake MD, Ansel GM, Jaff MR, et al., Zilver PTX Investigators. Durable clinical effectiveness with paclitaxel-eluting stents in the femoropopliteal artery: 5-year results of the Zilver PTX randomized trial. Circulation 2016;133: $1472-83$.

15. Axel DI, Kunert W, Göggelmann C, et al. Paclitaxel inhibits arterial smooth muscle cell proliferation and migration in vitro and in vivo using local drug delivery. Circulation 1997;96:636-45.

16. Stettler C, Wandel S, Allemann S, et al. Outcomes associated with drug-eluting and bare-metal stents: a collaborative network meta-analysis. Lancet 2007;370:937-48.

17. Abal M, Barasoain JM. Taxanes: microtubule and centrosome targets, and cell cycle dependent mechanisms of action. Curr Cancer Drug Targets 2003;3:193-203.

18. Horwitz S, Cohen D, Rao S, et al. Taxol: mechanisms of action and resistance. J Natl Cancer Inst Monogr 1993;15: $55-61$.

19. Dake MD, Ansel GM, Jaff MR, et al., Zilver PTX Investigators. Sustained safety and effectiveness of paclitaxeleluting stents for femoropopliteal lesions: 2-year follow-up from the Zilver PTX randomized and single-arm clinical studies. J Am Coll Cardiol 2013;61:2417-27.

20. Lepantalo M, Laurila K, Roth WD, et al., Scandinavian Thrupass Study Group. PTFE bypass or thrupass for superficial femoral artery occlusion? A randomised controlled trial. Eur J Vasc Endovasc Surg 2009;37:578-84.

21. Kedora J, Hohmann S, Garrett W, et al. Randomized comparison of percutaneous Viabahn stent grafts vs prosthetic femoral-popliteal bypass in the treatment of superficial femoral arterial occlusive disease. J Vasc Surg 2007;45: $10-6$. 